\title{
The Risk Prediction Model for Healthcare- Associated Infections among Elderly Patients Underwent Pancreaticoduodenectomy
}

\section{Yuejing Feng}

The Fifth Affiliated Hospital of Zhengzhou University

Hongwu Yao

Chinese PLA General Hospital

Jie Li

Shandong University

Mingmei Du

Chinese PLA General Hospital

\section{Xibao Gao}

Shandong University

Yunxi Liu

Chinese PLA General Hospital

Chengchao Zhou ( $\nabla$ zhouchengchao@sdu.edu.cn )

Shandong University

\section{Research Article}

Keywords: Healthcare-associated infections, Elderly, Pancreaticoduodenectomy, Risk prediction

Posted Date: January 15th, 2021

DOl: https://doi.org/10.21203/rs.3.rs-138219/v1

License: (c) (1) This work is licensed under a Creative Commons Attribution 4.0 International License.

Read Full License 


\section{The Risk Prediction Model for Healthcare-Associated Infections among Elderly Patients Underwent Pancreaticoduodenectomy}

Yuejing Feng ${ }^{1,2, \#, ~ H o n g w u ~ Y a o ~}{ }^{3, \#}$, Jie Li $^{2}$, Mingmei Du ${ }^{3}$, Xibao Gao ${ }^{2}$, Yunxi Liu ${ }^{3, *}$ and Chengchao Zhou $2,4, *$

1 The Fifth Affiliated Hospital of Zhengzhou University, Zhengzhou, 450052, China;

fengyuejing1994@163.com

2 School of Public Health, Shandong University, Jinan, 250012, China;

fengyuejing1994@163.com; lijie2@126.com; chem@sdu.edu.cn; zhouchengchao@sdu.edu.cn

3 Department of Disease Control and Prevention, The First Medical Center, Chinese PLA General Hospital, Beijing 100039, China;

arenewu@163.com; dumingm@163.com; liuyunxi301@qq.com

4 NHC Key Laboratory of Health Economics and Policy Research (Shandong University), Jinan 250012, China; zhouchengchao@sdu.edu.cn

\# These authors contributed equally to this work.

* Correspondence: liuyunxi301@qq.com; Tel: (+86) 0106693 7144(YX,L)

zhouchengchao@sdu.edu.cn; Tel: (+86) 5318838 1567(CC,Z) 


\begin{abstract}
Background: The elderly are a high-risk group of healthcare-associated infections (HAIs) after pancreatico duodenectomy (PD), and the effective prediction model may be beneficial to HAIs control.

Methods: The data were obtained from Hospital Infection Surveillance System. The BP-ANN model was conducted according to univariate analysis. The receiver operating characteristic curve (ROC), the prediction accuracy, sensitivity and specificity were used to estimate the predicted performance. The final weight coefficients were calculated to illustrate the relative importance of indicators.

Results: Of 688 elderly patients underwent PD, 83 (12.06\%) were diagnosed with HAIs. 9 significant factors $(\mathrm{P}<0.05)$ including weight, fever, continuous fever for more than three days, blood routine abnormal percentage, ever livered in intensive care unit (ICU), antibacterial combination, postoperative antibacterial use days, ventilator use and urinary catheter use days were included into prediction model. The prediction accuracy in testing sets was $93.79 \%$, and the sensitivity and specificity were 0.67 and 0.97 . The contribution level of 9 significant factors were $10.65 \%, 8.54 \%, 10.17 \%, 9.64 \%, 9.26 \%, 10.02 \%, 12.53 \%, 11.90 \%$ and $17.29 \%$, respectively.

Conclusions: The 9-9-1 BP-ANN prediction model underpinned by complex factors in this study has relatively excellent performance for HAIs prediction among elderly patients after PD. Urinary catheter use days, postoperative antibacterial use days, ventilator use, weight and continuous fever for more than three days are the top five contribution indicators for HAIs prediction, which should be fully taken into consideration when developing HAIs prediction for the PD patients.
\end{abstract}

Keywords: Healthcare-associated infections; Elderly; Pancreaticoduodenectomy; Risk prediction 


\section{Background}

Pancreaticoduodenectomy (PD), a complicated operation for the treatment of pancreatic cancer, ampullary carcinoma, pancreatic head disease, and other related diseases [1], has been widely applied in clinical practice with the increasing incidence of pancreatic cancer worldwide over the past decades, especially in developing countries [2]. PD may not only remove the gallbladders, but also clean up the relevant organs and tissues, thus increasing the risks of various postoperative complications [3-5]. Although the mortality rate of patients after PD has dropped down to below 5\% due to the development of medical technology, the incidence of postoperative complications remains high [6-9]. As one of the most common adverse events, healthcare-associated infections (HAIs) accounts for one-third of postoperative complications of PD [4], of which incidence ranges from $20.0 \%$ to $60.0 \%$, resulting in decreasing surgery success rate, lowering medical quality, and increasing medical cost and risk of mortality [10-14]. Previous studies have found that older patients underwent PD were of higher risk to be more susceptible to HAIs because of the decline of the function of various organs and immune [15-21]. Therefore, HAIs among the elderly patients after PD has become a prominent clinical event needed to pay more attention.

The risk prediction model underpinned by complex factors has been used to assess the occurrence risk of HAIs and other adverse effects on prognosis. Numerous studies suggested that providing risk prediction through comprehensive analysis of related factors was an effective measure to prevent adverse events [22-24]. A study on the prediction of morbidity after PD suggested that the mean muscle attenuation on routine preoperative CT scans seemed to be an indicator to predict postoperative complications [25]. Similarly, C-reactive protein was recognized as a reliable indicator for early postoperative prediction of pancreatic fistula after PD [26]. Joliat GR et al. applied the Braga score to calculate the overall risk score for the prediction of complications after PD [27]. A risk scoring system with 10-point based on logistic regression was also established to predict the pancreatic fistula [28]. However, most of studies mainly focused on general age groups, and just described one or more postoperative complications rather than targeting HAIs. And so far, few studies have prospectively explored the risk prediction of HAIs among patients after PD.

A systematic review pointed out that artificial neural network (ANN) is undoubtedly more advantageous than traditional statistical methods in predicting clinical outcomes when the relationship between outcome-related variables is complex, multi-dimensional and non-linear [34]. And its practicality has been verified among lung cancer patients, blood disease patients, and ICU patients [32, 35-36]. As one of the most common artificial neural networks, the back propagation artificial neural network (BP-ANN), based on the simulation of biological neural network, has also been proven to acquire an excellent performance in predictive model construction, of which prominent advantage is that it is unnecessary to determine the mathematical equation of the mapping relationship between input and output variables in advance, with the actual output results gradually approaching to the expected output value by error propagation [29-31]. Nowadays, BP-ANN has been widely applied into various complex systems and model constructions that contain multiple and non-linear or unclear relationship variables [32-33], which may be also suitable for predicting the HAIs underpinned by complex factors among patients after PD. Consequently, this study aims to construct a risk prediction model for HAI occurrence among elderly patients after PD using BP-ANN model, and to verify its predictive performance.

\section{Methods}

\section{Data sources}

The data used in this study was from a 3800-bed tertiary hospital with 135 wards serving approximately 13000 inpatients per month in Beijing, China. We collected the patient data using the automatic Hospital Infection Surveillance System (RT-NISS), which was developed since 2010 and put into practice since 2012 [37]. The RT-NISS automatically collected process data from the hospital information system and monitored all patients during their hospital stay. The related information of patients including the common information, diagnosis and therapy information, medication information, operation information and so on, could be directly obtained from the RT-NISS. The exported data were also confirmed by the infection prevention professionals. For protecting the privacy of the patients, all sensitive patient information in this study was excluded. Ethical approval was obtained from the Medical Ethics Committee of the Chinese PLA General Hospital (approval number S2019-142-02) and the informed consent was obtained from all subjects. 


\section{Inclusion criteria}

The eligibility of elderly patients after PD for this study was determined according to the following inclusion criteria: 1) Elder patients were 60 years old and over; 2) Patients admitted between 1 January 2014 and 31 December 2017; 3) Main surgical operation of patients was PD; 4) Patients had no HAI or occurred HAI before PD. Finally, a total 688 elderly patients underwent PD were screened out to meet the inclusion criteria in this study.

\section{HAI case definition}

The HAI cases were confirmed by infection preventers according to the Chinese Nosocomial Infection Diagnosis Criterion (2001) published by the National Health Commission of the People's Republic of China [38], which differed somewhat from the United States CDC Definitions for Nosocomial Infection (1988).

\section{Variables management}

The dependent variable was the HAI occurred or not, the infection cases were coded as 1, while non-infection cases were coded as 0 . Available data concerning demographic, admission and other factors was collected and used as dependent variables to explore potential determinants of the occurrence risk of HAIs among elderly patients after PD. Then 18 complete independent variables were identified as following parts: 1) Demographic characteristics: age, sex and weight; 2) Admission information: admission year, fever, continuous fever for more than three days, blood routine abnormal percentage; operation duration, preoperative hospital stay and ever lived in ICU; 3) Underlying disease: hypertension, diabetes, and coronary heart disease; 4) Antibacterial utilization: preoperative antibacterial use, antibacterial combination and postoperative antibacterial use days; 5) Medical device utilization: ventilator use and urinary catheter use days. Specifically, all the variables in HAI group including fever, continuous fever for more than three days, blood routine abnormal percentage and others were collected before the diagnosis of HAI. Furthermore, the low-quality and incomplete variables of central venous catheter use, urinary catheter use, ventilator and central venous catheter use days in medical device utilization part were excluded in this study.

\section{Data analysis}

The descriptive statistics were performed, of which continuous variables was presented as means with standard deviation or median with quartile, and categorical variables as frequencies and proportions. Univariate analysis using Chi-square tests, two sample t-tests and Wilcox rank sum test was performed to examine the effect of each individual variable and identify potential indicative factors of HAIs among elderly patients after PD, and the statistical significance was set at the $5 \%$ level.

Then a three-layer BP-ANN model containing statistically significant variables in univariate analysis was constructed. The data from 2014-2016 was used for training set while the data from 2017 for testing set, leading to 527 patients in training set and 161 patients in testing set, respectively. The number of hidden layer of the model was selected referring to the Equation 1:

$$
\mathbf{m}=\sqrt{\boldsymbol{n}+\boldsymbol{l}}+\mathbf{a}
$$

where $\mathbf{m}$ is the number of hidden layer nodes, $\boldsymbol{n}$ is the number of input layer nodes (the number of significant variables included in the model), $\boldsymbol{l}$ is the number of output layer nodes (the number of outcomes, $\boldsymbol{l}=\mathbf{1}$ in this study), and $\mathbf{a}$ is a constant between 1 and 10 .

The weight coefficients were calculated referring to the Equation 2.

$$
I_{j}=\frac{\sum_{m=1}^{N h}\left(\left(\left|\omega_{j m}^{1}\right| / \sum_{k=1}^{N i}\left|\omega_{k m}^{1}\right|\right) \times\left|\omega_{m n}^{2}\right|\right)}{\left.\sum_{k=1}^{N i}\left\{\sum_{m=1}^{N h}\left(|| \omega_{j m}^{1}\left|/ \sum_{k=1}^{N i}\right| \omega_{k m}^{1} \mid\right) \times\left|\omega_{m n}^{2}\right|\right)\right\}}
$$


where $\boldsymbol{I}_{\boldsymbol{j}}$ represents the weight coefficient of the j input variable to the output, $\boldsymbol{N i}$ and $\boldsymbol{N h}$ are the number of input and hidden layer nodes, $\boldsymbol{\omega}^{\mathbf{1}}$ is the connect weight values of input-hidden layer and $\boldsymbol{\omega}^{\mathbf{2}}$ is connect weight values of hidden-output layer. $\boldsymbol{j}, \boldsymbol{m}$ and $\boldsymbol{n}$ are the serial number of the neuron.

Sigmoid transfer function was employed as an excitation function between the hidden layer and the output layer. The initial learning rate was set 0.1 and the training goal was set 0.0001 . The BP-ANN model was trained with 5000 epochs, and the prediction threshold was set 0.5 .

Finally, in order to verify the prediction performance of the model for the testing set, the overall prediction accuracy, sensitivity, specificity and the area under the receiver operating characteristic (ROC) curve (AUC) were also calculated. The weight coefficients were displayed to determine the contribution level of significant variable for HAIs prediction among elderly patients after PD.

Descriptive and univariate analyses were performed in STATA14.0. The BP-ANN prediction model was constructed in MatlabR2014a.

\section{Results}

Of 688 elderly patients after PD, 426 (61.92\%) were males and $262(38.08 \%)$ were females. The mean age was 66.685 .12 years and the average hospital stay was $25.23 \quad 9.69$ days. There were $7.70 \%, 6.98 \%$, and $2.18 \%$ of patients with hypertension, diabetes, and coronary heart disease, respectively. 83 patients developed HAIs with the overall prevalence of $12.06 \%$ (see Table 1). The annual prevalence of HAI from 2014 to 2017 was $14.91 \%, 16.07 \%, 9.84 \%$ and $9.26 \%$, respectively. For patients with HAIs, $40.96 \%$ were postoperative abdominal infections $(34,40.96 \%)$, followed by bloodstream infections $(11,13.25 \%)$, surgical site infections $(9,10.84 \%)$, lower respiratory tract infections $(8,9.64 \%)$, organ cavity infections $(5,6.02 \%)$ and others $(13,15.68 \%)$ (see Table 2).

Table 1 Basic information of the participants $(\mathrm{N}=688)$

\begin{tabular}{lcc}
\hline Variables & $\mathbf{n}$ & Percentage (\%) \\
\hline Age, years $(\overline{\mathrm{x}} \pm \mathrm{s})$ & $66.68 \pm 5.12$ & - \\
Weight, $\mathrm{kg}(\overline{\mathrm{x}} \pm \mathrm{s})$ & $64.02 \pm 11.32$ & - \\
Hospitalization days $(\overline{\mathrm{x}} \pm \mathrm{s})$ & $25.23 \pm 9.69$ & 61.92 \\
Sex & & 38.08 \\
$\quad$ male & 426 & \\
$\quad$ female & 262 & 16.57 \\
Admission year & & 24.42 \\
$\quad 2014$ & 114 & 35.47 \\
2015 & 168 & 23.55 \\
$\quad 2016$ & 244 & \\
$\quad 2017$ & 162 & 7.70 \\
Hypertension & & 92.30 \\
$\quad$ yes & 53 & 6.98 \\
$\quad$ no & 635 & 93.02 \\
Diabetes & & 2.18 \\
$\quad$ yes & 40 & 97.82 \\
$\quad$ no & 640 & \\
Coronary heart disease & & \\
$\quad$ yes & 15 & \\
no & 673 & \\
\hline HAI & & \\
\hline
\end{tabular}




\begin{tabular}{lcc}
\hline yes & 83 & 12.06 \\
no & 605 & 87.94 \\
\hline
\end{tabular}

Table 2 The information of patients with HAIs $(\mathrm{Ni}=83)$

\begin{tabular}{llc}
\hline Variables & n & Percentage (\%) \\
\hline Admission year & & \\
2014 & 17 & 14.91 \\
2015 & 27 & 16.07 \\
2016 & 24 & 9.84 \\
2017 & 15 & 9.26 \\
Infection type & & \\
postoperative abdominal infections & 37 & 44.58 \\
bloodstream infections & 11 & 13.25 \\
surgical site infections & 9 & 10.84 \\
lower respiratory tract infections & 8 & 9.64 \\
$\quad$ organ cavity infections & 5 & 6.02 \\
$\quad$ other infections & 13 & 15.68 \\
\hline
\end{tabular}

Univariate analysis was performed to examine the effect of variables screening for risk prediction model, which had identified altogether 9 significant factors associated with the occurrence of HAIs. In demographic characteristics part, the average weight of patients who developed HAIs $\left(\begin{array}{lll}66.10 & 10.83 \mathrm{~kg}\end{array}\right)$ was greater than that of non-HAIs (63.74 $0.46 \mathrm{~kg})$. As for admission information part, the fever, continuous fever for more than three days, blood routine abnormal percentage and ever lived in ICU were found as significant variables. In addition, the prevalence of HAIs among patients taking antimicrobials in combination (20.42\% vs. 6.19\%) and using ventilator (47.19\% vs. $6.84 \%$ ) was higher, and the HAI group had significantly longer days of postoperative antibacterial use and urinary catheter use than those of non-HAI group (see Table 3 ).

Table 3 Univariate analysis of factors associated with HAIs (N=688)

\begin{tabular}{|c|c|c|c|c|}
\hline \multirow[b]{2}{*}{ Variables } & \multicolumn{2}{|c|}{ Infection status } & \multirow[b]{2}{*}{$\chi^{2} / \mathbf{t} / z$} & \multirow[b]{2}{*}{$P$-value } \\
\hline & $\begin{array}{c}\text { Infection } \\
\text { n }(\%)\end{array}$ & $\begin{array}{c}\text { Non-infection } \\
\text { n }(\%)\end{array}$ & & \\
\hline \multicolumn{5}{|c|}{ Demographic Characteristics } \\
\hline Age, years $(\bar{x} \pm s)$ & $66.90 \pm 5.30$ & $66.65 \pm 5.10$ & -0.426 & 0.670 \\
\hline Weight, $\operatorname{kg}(\bar{x} \pm s)$ & $66.10 \pm 10.83$ & $63.74 \pm 0.46$ & -1.784 & 0.038 \\
\hline Sex & & & 1.234 & 0.267 \\
\hline male & $56(13.15)$ & $370(86.85)$ & & \\
\hline female & $27(10.31)$ & 235 (89.69) & & \\
\hline \multicolumn{5}{|l|}{ Admission Information } \\
\hline Admission year & & & 5.758 & 0.124 \\
\hline 2014 & $17(14.91)$ & $97(85.09)$ & & \\
\hline 2015 & $27(16.07)$ & $141(83.93)$ & & \\
\hline 2016 & $24(9.84)$ & $220(90.16)$ & & \\
\hline 2017 & $15(9.26)$ & $147(90.74)$ & & \\
\hline Fever & & & 53.827 & $<0.01$ \\
\hline
\end{tabular}


yes

no

Continuous fever for more than

three days

$$
\text { yes }
$$

no

\section{Operation duration}

$$
\mathrm{M}\left(\mathrm{P} 2{ }_{5}, \mathrm{P}_{75}\right) \#
$$

Blood routine abnormal percent age $\mathrm{M}\left(\mathrm{P}_{25}, \mathrm{P}_{75}\right) \#$

Preoperative hospital stay

$$
\mathrm{M}\left(\mathrm{P}_{25}, \mathrm{P}_{75}\right) \#
$$

Ever lived in ICU

$$
\text { yes }
$$

no

\section{Underlying Disease}

Hypertension

$$
\text { yes }
$$

no

Diabetes

$$
\text { yes }
$$$$
\text { no }
$$

Coronary heart disease

$$
\text { yes }
$$

no

\section{Antibacterial Utilization}

Preoperative antibacterial use

$$
\text { yes }
$$$$
\text { no }
$$

\section{Antibacterial combination}

$$
\text { yes }
$$

no

Postoperative antibacterial use days $\mathrm{M}\left(\mathrm{P} 2_{5}, \mathrm{P}_{75}\right) \#$

\section{Medical Device Utilization}

Ventilator use

$17(50.00)$

$66(10.09)$

$5.14(4.33,6.07)$

$31.25(22.22,37.50)$

$7(5,11)$

8 (47.06)

75 (11.18)$$
9(52.94)
$$

596 (88.82)

17 (50.00)

$588(89.91)$

$5.08(4.17,5.99)$

$-0.960$

0.337

$$
27.27(16.13,37.50)
$$

$-2.090$

0.037

$7(5,10)$

0.480

0.631

20.122

$<0.01$

6 (11.32)

77 (12.13)

5 (10.42)

78 (12.19)

2 (13.33)

81 (12.04)

2.141

0.143

67 (13.14)

$16(8.99)$

58 (20.42)

25 (6.19)

$17(13,21)$

443 (86.86)

162 (91.01)

31.852

$<0.01$

226 (79.58)

379 (93.81)

$$
11(8,13)
$$

$-8.719$

$<0.01$

118.899

$<0.01$

$$
\begin{gathered}
42(47.19) \\
41(6.84)
\end{gathered}
$$$$
47 \text { (52.81) }
$$

$558(93.16)$

$$
2(2,3)
$$

$-12.079$

$<0.01$ $\mathrm{M}\left(\mathrm{P}_{25}, \mathrm{P}_{5}\right)$ \#

${ }^{\#}$ M means the median of non-normally distributed data, P25 and P75 means the 25th quantile and the 75th quantile of data. 
According to the formula of hidden layer nodes selection, 5-14 was the range of the number of hidden layer nodes. Finally, 9 hidden layer nodes with the highest prediction accuracy and the shortest training time in the training set was selected in this study, then the three-layer 9-9-1 BP-ANN framework was employed to predict the HAIs of elderly patients after PD in the testing set. The results showed that the ultimate prediction accuracy was $93.79 \%$, and the sensitivity and specificity of the BP-ANN were 0.67 and 0.97 , respectively. See Additional file:Figure $\mathrm{S} 1$ for the visualize prediction results of the model. Furthermore, in order to examine the prediction performance of the model, the ROC was drawn with AUC of 0.94 (see Figure 1).

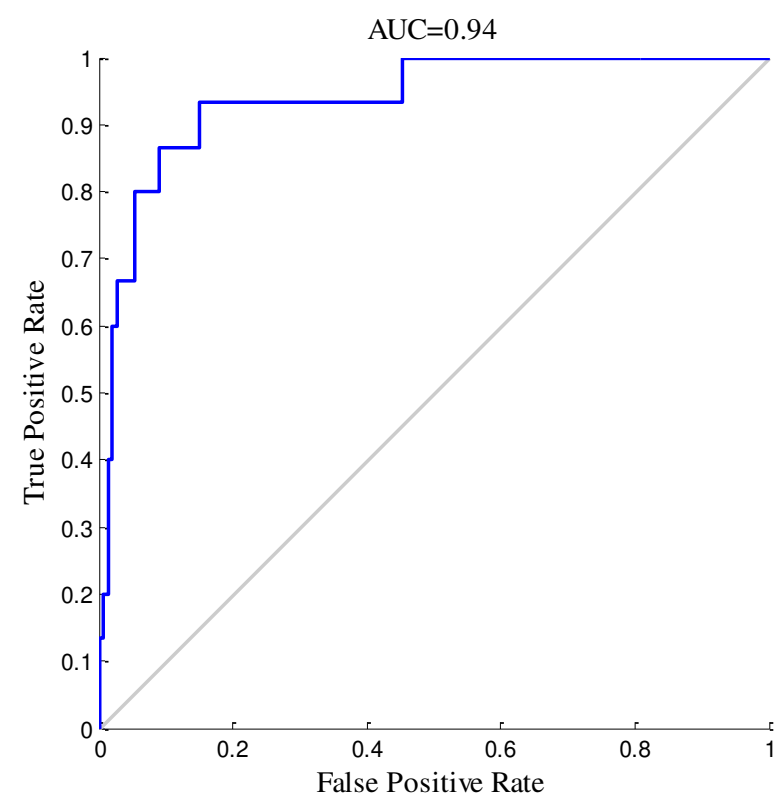

Figure1 Receiver operating characteristic curves of BP-ANN model

After calculating the weight coefficient, urinary catheter use days $(I=17.29 \%)$ is of the largest contribution value, which together with postoperative antibacterial use days $(I=12.53 \%)$, ventilator use $(I=11.90 \%)$, weight $(I=10.65 \%)$ and the continuous fever for more than three days $(I=10.17 \%)$ constitute the top five contribution indicators for HAIs occurrence prediction (see Figure 2). See Additional file:Table S1 for all the connection weights values of the model between input-hidden layer and hidden-output layer.

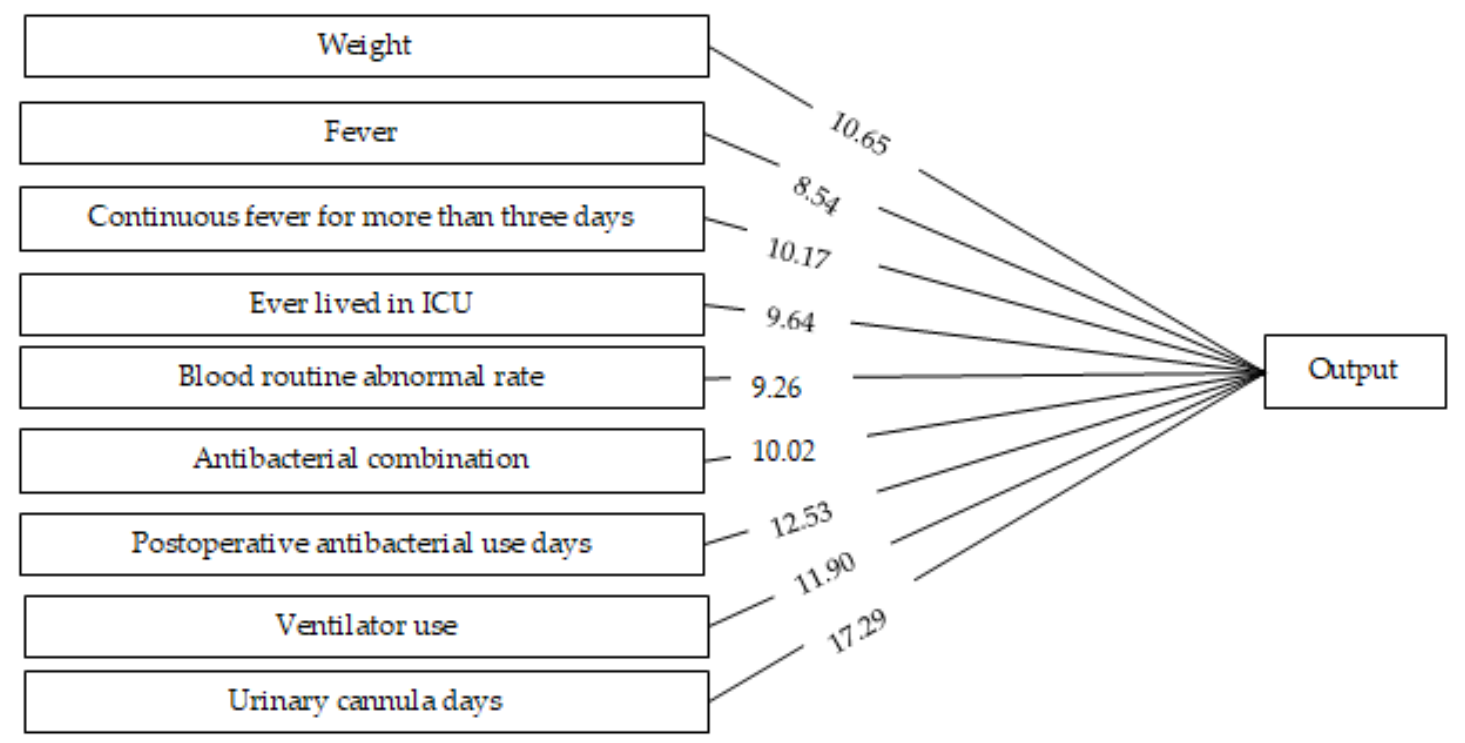

Figure 2 Relative weight coefficient of input variables (\%) 


\section{Discussion}

In the current study, the overall prevalence of HAIs among elderly patients after PD was $12.06 \%$, which was lower than the reported infection rate of $17.80 \%$ in Dalian, China, while higher than $8.12 \%$ among PD patients aged 61-83 years old in Chun'an, Zhejiang, China [39-40]. The differences may result from the different research settings and participants. It implies that the HAI prevalence of the elderly patients after PD in this study was at a relatively moderate level, and the further improvement of prevention and control measures was needed.

Based on the univariate analysis, the risk prediction model using BP-ANN involving 9 significant factors for predicting HAI occurrence among elderly PD patients was finally constructed. The overall prediction accuracy of the model, $93.79 \%$, was obviously higher than the reported predictive rate of $75.7 \%$ conducted in lung cancer patients [32] and $82.49 \%$ conducted among hematological diseases patients [41], and also higher than the reported predictive performance conducted by 10-point Braga risk score among patients underwent PD [28]. It had indicated that BP neural network prediction accuracy could reach approximately $90 \%$ by scientifically determining risk indicators and constructing appropriate network models [42], which was well verified in this study. The sensitivity of the risk prediction model in this study was 0.67 while the specificity was 0.97 , which were also both higher than other similar studies [32, 41]. Furthermore, the AUC value of 0.94 was higher than the study among lung cancer patients conducted by Chen et al [32]. These all indicated that our BP-ANN model has an excellent goodness-of-fit for HAIs prediction among elderly PD patients.

From the final weights coefficient result in the model, we found that medical device indwelling including urinary cannula days and ventilator use were two key indicators with the high contribution weights. The use and built-in of medical devices increase the risk of device-related HAIs, which can deteriorate the extent of infections due to surgery. Many studies had pointed out that catheter-associated urinary tract infection was correlated with duration of catheterization and suggested to shorten the length of catheter indwelling [43-44]. A previous study estimated that ventilator-associated infection was associated with the application of mechanical ventilation [52]. It implies that the patients indwelling medical devices can be of high risk of HAIs.

Furthermore, postoperative antibacterial use days were identified as the second contributing indicator for HAIs prediction among elderly patients underwent PD. Though antibiotic use has irreplaceable clinical value in the prevention of clinical HAIs, clinicians should still choose the appropriate timing of medication, duration of medication and type of medication to ensure rational use of drugs, avoiding drug resistance and other adverse events caused by antibiotic abuse [45-47]. The correlation between antimicrobial use and HAI occurrence should be emphasized and monitored in the future.

We also found that continuous fever for more than three days was one of the top five important predictors. HAIs and fever symptoms could interact with each other, that is, long-term fever can caused HAIs meanwhile patients with HAIs could exacerbate fever. Furthermore, prolonged fever days might cause the immunity to decline, thus making patients be more susceptible to HAIs. Some studies have also pointed out that the incidence of HAIs in patients with prolonged fever was higher than that of ordinary patients [48]. This finding indicated that long-term fever was the key factor that cannot be ignored both in clinical and HAI prediction. What's more, weight was the fifth contributing indicator in the model. Huttunen R. pointed out that obesity increased the risk of HAIs and the health management of weight could effectively prevent its occurrence [49]. The effect of weight on surgical site infections had also been confirmed in many other studies [50-51]. So we speculate that patients with underweight might have low immunity and patients with overweight might have weight-related diseases, which are both associated with HAIs in elderly PD patients.

This study is the first to apply the BP-ANN model underpinned by complex factors to HAI occurrence prediction among elderly patients after PD. However, this study has also several limitations. First, it was difficult for us to include all the HAIs-related variables in the analysis due to the characteristics of clinical data, and the personal socioeconomic status information in this study only includes age, gender and weight, which may miss partly important information. Secondly, only one hospital's data was selected for prediction model construction, which may result in selective bias. Thirdly, the BP-ANN prediction model was constructed among elderly PD patients, so that the ability to extrapolate to the general hospitalized patients may be limited.

\section{Conclusions}

In this study, we have constructed a 9-9-1 BP-ANN risk prediction model for HAIs among elderly patients after PD, and it was proved to have a good predictive performance. Nine indicative variables screened in the risk prediction model can provide a balanced indication, so it can be promotionally applied into clinical HAIs 
prediction for elderly patients after PD. And the factors including the urinary catheter use days, postoperative antibacterial use days, ventilator use, weight and continuous fever for more than three days were the top five contributed indicators, which should be fully taken into consideration when developing HAIs prediction and surveillance system for the PD patients.

\section{Ethics approval and consent to participate}

This study was approved by the Medical Ethics Committee of the Chinese PLA General Hospital (approval number S2019-142-02). All methods were performed in accordance with the relevant guidelines and regulations.

\section{Consent for publication}

The manuscript is approved by all authors for publication.

\section{Availability of data and materials}

All data concerning this study is included in this published article.

\section{Competing interests}

The authors declare that they have no competing interests.

\section{Funding}

This research was funded by the Special Program for Prevention and Control of Infectious Diseases in China [grant number 2018ZX10733-402], the National Key R\&D Program of China [grant number 2017YFC0806308], and the Medical Youth Program of Chinese PLA General Hospital [grant number QNF19044].

\section{Authors' contributions}

Conceptualization, Yuejing Feng, Hongwu Yao, Yunxi Liu and Chengchao Zhou; Data curation, Jie Li; Formal analysis, Yuejing Feng; Funding acquisition, Mingmei Du and Yunxi Liu; Investigation, Hongwu Yao and Xibao Gao; Methodology, Yuejing Feng and Chengchao Zhou; Project administration, Yunxi Liu; Resources, Hongwu Yao, Mingmei Du, Xibao Gao, Yunxi Liu and Chengchao Zhou; Software, Yuejing Feng; Supervision, Hongwu Yao, Jie Li, Mingmei Du, Xibao Gao and Chengchao Zhou; Validation, Yunxi Liu; Writing - original draft, Yuejing Feng; Writing - review \& editing, Hongwu Yao, Jie $\mathrm{Li}$ and Chengchao Zhou.

\section{Acknowledgements}

Not applicable 


\section{References}

1. Gerstenhaber F, Grossman J, Lubezky N, Itzkowitz E, Nachmany I, Sever R, et al. Pancreaticoduodenectomy in Elderly Adults: Is It Justified in Terms of Mortality, Long-Term Morbidity, and Quality of Life? J Am Geriatr Soc 2013; 61:1351-7.

2. Dudeja V, Livingstone A. Is age just a number: pancreaticoduodenectomy in elderly patients? Hepatob Pancreat Dis 2016; 15:346-7.

3. Xuelu Li, Dandan Zhu, Chunming Yang, Zuowei Zhao. New progress in minimally invasive pancreaticoduodenectomy. Chin J General Surgery 2015; 24:402-7.

4. Juhong Yang. Risk factors analysis and protective countermeasures of infections after pancreatoduodenectomy. Nursing Practice and Research 2017; 14:100-4.

5. Naimin Shi, Yong Li, Shuzhen Lv, Cuicui Lv. Analysis of the related factor of complications in patients treated with laparoscopic pancreaticoduodenectomy. J Bengbu Med Coll 2018; 43:216-8.

6. Kawai M, Yamaue H. Analysis of clinical trials evaluating complications after pancreaticoduodenectomy: A new era of pancreatic surgery. Surg Today 2010.40:1011-7.

7. Hangbao Li, Jun Zhou, Fengqing Zhao, Yifan Lu. A Meta-analysis of risk factors of postoperative infectious complications after pancreatoduodenectomy. J Hepato Surg 2017; 29:215-9.

8. Hall BL, Hirbe M, Yan Y, Khuri SF, Henderson WG, Hamilton BH. Thyroid and Parathyroid Operations in Veterans Affairs and Selected University Medical Centers: Results of the Patient Safety in Surgery Study. J Am Coll Surgeons 2007; 204:1222-34.

9. Yasuhiro Ito, Takeshi Kenmochi, Tomoyuki Irino, Tomohisa Egawa, Shinobu Hayashi,Atsushi Nagashima, Yuko Kitagawa. The impact of surgical outcome after pancreaticoduodenectomy in elderly patients. World J Surg Onc 2011; 9:102.

10. K. Okano THMU. Postoperative infectious complications after pancreatic resection. Brit J Surg 2015; 102:1551-60.

11. Gouma DJ, van Geenen RCI, van Gulik TM, de Haan RJ, de Wit LT, Busch OR, Obertop H. Rates of Complications and Death After Pancreaticoduodenectomy: Risk Factors and the Impact of Hospital Volume. Annals of Surgery 2000; 232:786-794.

12. De Pastena M, Paiella S, Marchegiani G, Malleo G, Ciprani D, Gasparini C, Secchettin E, Salvia R, Bassi C. Postoperative infections represent a major determinant of outcome after pancreaticoduodenectomy: results from a high-volume center. Surgery 2017; 162: 792-801.

13. TS Kent, , TE Sachs, MP Callery, CM Vollmer Jr. The burden of infection for elective pancreatic resections. Surgery 2013; 153:86-94.

14. Khan HA, Baig FK, Mehboob R. Nosocomial infections: Epidemiology, prevention, control and surveillance. Asian Pacific Journal of Tropical Biomedicine 2017; 7:478-82.

15. Shin JW, Ahn KS, Kim YH, Kang KJ, Lim TJ. The impact of old age on surgical outcomes after pancreaticoduodenectomy for distal bile duct cancer. Korean J Hepatobiliary Pancreat Surg 2011; 15:248-53.

16. Makary MA, Winter JM, Cameron JL, Campbell KA, Chang D, Cunningham SC, Riall TS, Yeo CJ. Pancreaticoduodenectomy in the Very Elderly. J Gastrointest Surg 2006; 10:347-56.

17. Shigang Duan, Ping Chen, Ying Li, Jiming Ding, ZHANG Lin. Factors Associated with Fungal Infection Following Pancreatoduodenectomy. Chin J Bases Clin General Surg 2006; 13:317-20.

18. Riall TS, Reddy DM, Nealon WH, Goodwin JS. The effect of age on short-term Outcomes after pancreatic resection: a population-based study. Ann Surg 2008; 248:459-467.

19. Lightner AM, Glasgow RE, Jordan TH, Krassner AD, Way LW, Mulvihill SJ, Kirkwood KS. Pancreatic resection in the elderly. J Am Coll Surg 2004; 198:697-706.

20. McPhee JT, Hill JS, Whalen GF, Zayaruzny M, Litwin DE, Sullivan ME, Anderson FA, Tseng JF. Perioperative mortality for pancreatectomy: a national perspective. Ann Surg 2007; 246:246-253.

21. Haigh P I, Bilimoria K Y, DiFronzo L A. Early postoperative outcomes after pancreaticoduodenectomy in the elderly. Archives of Surgery 2011; 146: 715-723.

22. Reese SM, Knepper B, Young HL, Mauffrey C. Development of a surgical site infection prediction model in orthopaedic trauma: The Denver Health Model. Injury 2017; 48:2699-704.

23. Haley RW, Culver DH, White JW, Morgan WM, Emori TG, Munn VP, Hooton TM. The efficacy of infection surveillance and control programs in preventing nosocomial infection in US hospitals. Am J Epidemiol 1985; 121:182-205. 
24. Maruthappu M, Trehan A, Barnett-Vanes A, McCulloch P, Carty MJ. The Impact of Feedback of Surgical Outcome Data on Surgical Performance: A Systematic Review. World J Surg 2015; 39:879-89.

25. Nicolas Linder, Alexander Schaudinn, Katharina Langenhan, Felix Krenzien, Hans-Michael Hau, Christian Benzing, Georgi Atanasov, Moritz Schmelzle, Thomas Kahn, Harald Busse, Michael Bartels, Ulf Neumann, Georg Wiltberger. Power of computed-tomography-defined sarcopenia for prediction of morbidity after pancreaticoduodenectomy. BMC Med Imaging 2019; 19:32

26. Partelli S, Pecorelli N, Muffatti F, Belfiori G, Crippa S, Piazzai F, Castoldi R, Marmorale C, Balzano G, Falconi M. Early Postoperative Prediction of Clinically Relevant Pancreatic Fistula after Pancreaticoduodenectomy: usefulness of C-reactive Protein. HPB(Oxford) 2017; 19:580-586

27. Joliat GR, Petermann D, Demartines N, Schäfer M. Prediction of Complications After Pancreaticoduodenectomy Validation of a Postoperative Complication Score. Pancreas 2015; 44:1323-8.

28. Ye Li, Fang Zhou, Dongming Zhu, Zixiang Zhang, Jian Yang, Jun Yao, Yijun Wei, Yaling Xu, Deichun Li, Jian Zhou. Novel risk scoring system for prediction of pancreatic fistula after pancreaticoduodenectomy. World J Gastroenterology $2019 ; 25: 2650-2664$

29. Wang J, Wang F, Liu Y, Xu J, Lin H, Jia B, Zuo W, Jiang Y, Hu L, Lin F. Multiple linear regression and artificial neural network to predict blood glucose in overweight patients. Experimental and Clinical Endocrinology \& Diabetes 2016; 24: 34-38.

30. Ma J, Cai J, Lin G, Chen H, Wang X, Wang X, Hu L. Development of LC-MS determination method and back-propagation ANN pharmacokinetic model of corynoxeine in rat. J Chromatography B 2014; 959:10-5.

31. Wendong Liu, Ying Wu, Jing Ai, Qi Liang, Jianli Hu, Qigang Dai, Yuan Li, Fenyang Tang. Application of BP neural network in predicting the incidence of diarrhea. Chinese Journal of Health Statistics 2012; 29:801-4.

32. Chen J, Pan QS, Hong WD, Pan J, Zhang WH, Xu G, Wang YM. Use of an Artificial Neural Network to Predict Risk Factors of Nosocomial Infection in Lung Cancer Patients. Asian Pac J Cancer P 2014; 15:5349-53.

33. Cheng C, Cheng X, Dai N, Jiang X, Sun Y, Li W. Prediction of facial deformation after complete denture prosthesis using BP neural network. Comput Biol Med 2015; 66:103-12.

34. A. Bartosch-Harlid, B. Andersson, U. Aho, J. Nilsson, R. Andersson. Artificial neural networks in pancreatic disease. British Journal of Surgery. Incorporating European Journal of Surgery and Swiss Surgery 2008; 95: 817-826.

35. Duoshuang Xie, Qiao Hu, Rui Li, Qingqin Luo, Xiangyun Fu, Huifang Wang, Shaofa Nie. Construction of Artificial Neural Network Predictive Model for Hospital Infections. J Hbum 2015; 34:246-9.

36. Linyong Xu, Yi Bai, Ming Hu, Yongyong Xu, Zhen-qiu Sun. Artificial neural network in the prediction of nosocomial infection risk. J CentSouth Univ (Med Sci) 2006; 31:404-7.

37. Du M, Xing Y, Suo J, Liu B, Jia N, Huo R, Chen C, Liu Y. Real-time automatic hospital-wide surveillance of nosocomial infections and outbreaks in a large Chinese tertiary hospital. BMC Med Inform Decis Mak 2014; 9 (14).

38. The Ministry of Public Health: The nosocomial infections diagnosis criterion. Natl Med J China 2001; 81:314-320

39. Jin Xing, Dong Shang. Infection-related risk factor analysis after pancreaticoduodenectomy. J Dalian Medical University $2015 ; 37: 165-8$

40. Aihong Fang. The incidence of nosocomial infections and nursing countermeasures in elderly patients after pancreatoduodenectomy. Clinical Education of General Practice 2014; 12:358-9.

41. Libing Chen, Yunxi Liu, Mingmei Du, Yubin Xing, Jijiang Suo, Shengshan Cao. BP neural network in prediction of nosocomial infections in patients with hematological diseases. Chin Nosocomial 2014; 24:1542-4.

42. Zhi Wang, Wei Liu, Yan Lan. Study on the risk prediction model of nosocomial infection in cerebrovascular diseases based on BP neural network. Chongqing Medicine 2017; 46:1691-3.

43. Aixiang Yang, Huijuan Wu, Zhencang Zheng, Jing Yang, Ling Teng. Urinary Nosocomial Infection in Intensive Care Unit:A Clinical Analysis [J]. Chin J Epidemiol 2009; 19:1353-55.

44. Sugata Dasgupta, Soumi Das, Neeraj S. Chawan, Avijit Hazra. Nosocomial infections in the intensive care unit: Incidence, risk factors, outcome and associated pathogens in a public tertiary teaching hospital of Eastern India. Indian J Crit Care Med 2015; 19: 14-20.

45. Shuhui Wang, Wenhua Jing, Yingxia Li, Haiyan Wang. Risk factors and countermeasures of nosocomial infection in elderly patients in intensive care unit ( ICU). Chin J Public Health 2011;27:1570-1.

46. Zhao Z. Clinical application of antibiotics in prevention of general surgical incision infection. Clinical Medical Literature Electronic Journal 2019; 6:84-95.

47. Xiaomei $\mathrm{Wu}$. The value of rational use of antibiotics in the prevention of wound infection in general surgery. Clinical Rational Drug Use 2013; 6:14. 
48. Qiong Lu. Rational use of antibacterial drugs to reduce nosocomial infections. Chin J of Clinical Rational Drug Use 2015; $8: 174$.

49. Huttunen R, Karppelin M, Syrjänen J. Obesity and nosocomial infections. J Hosp Infect 2013; 85:8-16.

50. Dan Xing, Xinlong Ma, Jianxiong Ma, Jie Wang, Yang Chen, Yang Yang, Zhu Shaowen, Ma Baoyi, Feng Rui. A methodological, systematic review of evidence-based independent risk factors for surgical site infections after spinal surgery. Eur Spine J 2013; 22:605-15.

51. R.S.Namba, M.C.S.Inacio, E.W.Paxton. Risk factors associated with surgical site infection in 30491 primary total hip replacements. J Bone Joint Surg Br 2012; 94:1330-8.

52. Beardsley AL, Nitu ME, Cox EG, Benneyworth BD. An Evaluation of Various Ventilator-Associated Infection Criteria in a PICU. Pediatr Crit Care Med 2016; 17:73-8 
Figures

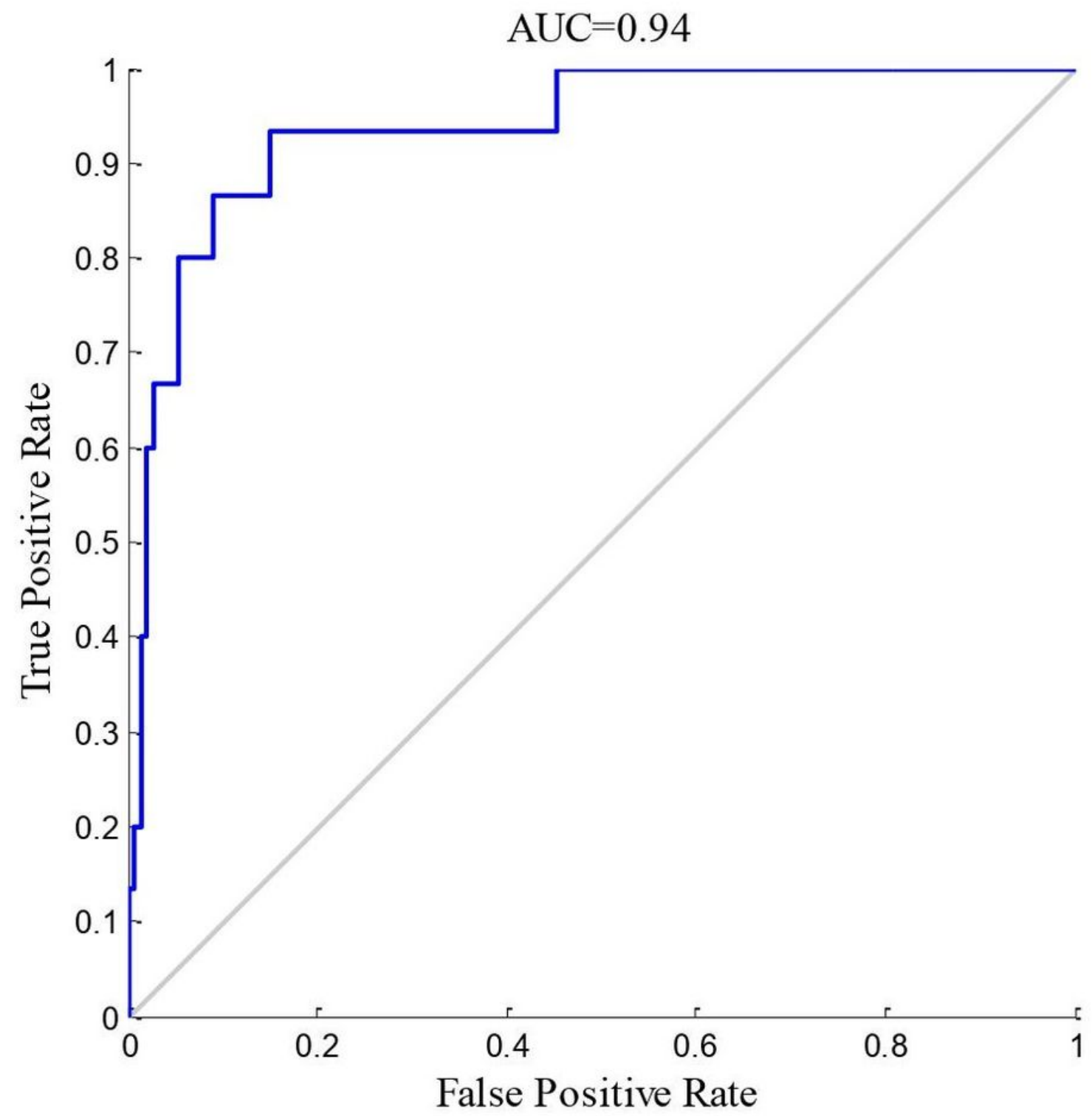

Figure 1

Receiver operating characteristic curves of BP-ANN model 


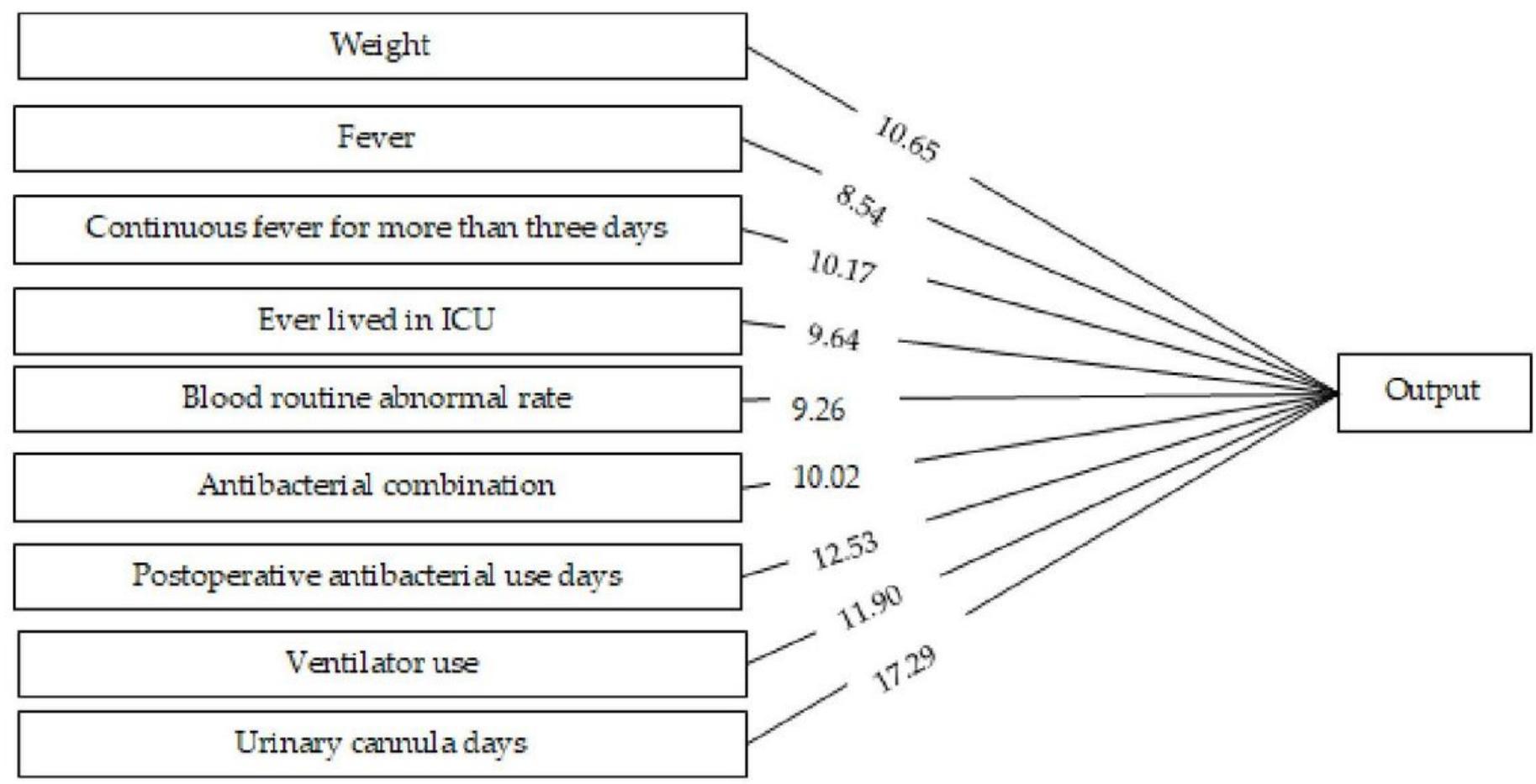

Figure 2

Relative weight coefficient of input variables (\%)

\section{Supplementary Files}

This is a list of supplementary files associated with this preprint. Click to download.

- Additionalfile.docx 